\title{
Comparison of Shear Design Models for Reinforced Concrete Beams Strengthened with Inorganic Matrix Composite Materials
}

\author{
Christos G. Papakonstantinou and Dimitris Bekiaris
}

\begin{abstract}
Externally bonded Fiber Reinforced Polymer (FRP) materials can be used to increase the shear strength of reinforced concrete (RC) structural members. These composite materials are typically based on organic matrices, which are used to impregnate the fibers and provide bond to the concrete surface. Existing analytical models were derived using experimental data from specimens strengthened with organic specimens. The aim of this study is to evaluate these models and verify if these models can be used also for specimens that utilize inorganic matrices. The methodology used in this study is based on a comparison of experimental values obtained from specimens strengthened with inorganic matrices to analytical values calculated using the aforementioned models. It is concluded, that most of the models for organic matrices can also be used for inorganic, but for a better correlation of results some adjustments are necessary.
\end{abstract}

Index Terms-Shear strengthening, FRP, fiber composites, rehabilitation, inorganic matrix.

\section{INTRODUCTION}

Many aging reinforced concrete structures can be considered obsolete or structurally deficient. Several different methods have been implemented in terms of strengthening these structures [1]-[3]. Some of them deal with concrete jackets that generally perform well, but result in an increase structural weight increase, which may create new problems especially in seismic regions. During the last years materials such as Fiber reinforced polymers (FRP) have been investigated for strengthening of existing structures [3]-[6]. These included mainly Glass and Carbon fibers impregnated with organic matrices. These composite materials were externally bonded to existing reinforced concrete elements such as beams, columns or slabs to provide flexural and/or shear strengthening. Later on several researchers started investigating other inorganic matrices such as Geopolymers [3], cementitious mortars [7], etc. The main target was to provide a better bond between the concrete substrate and FRP. It was shown in many studies that the main disadvantage of organic matrices was that only a portion of the FRP strength was utilized, since failure occurred by delamination of the FRP and not fracture of the fibers [8]. Almost all available analytical models and code provisions deal with the use of organic matrices in externally bonded FRP systems used for shear strengthening of reinforced concrete beams. The target of this paper is to

Mauscript received December 15, 2016; revised February 6, 2017.

Christos G. Papakonstantinou and Dimitris Bekiaris are with the Dept of Civil Engineering, University of Thessaly, Volos, Greece (e-mail: cpapak@uth.gr; dimitrisbekiaris@yahoo.com). investigate if existing models, derived using experimental data from specimens strengthened with organic matrix composites, can reliably predict the experimental shear strength of RC beams strengthened with inorganic matrix composites. In order to do so, an experimental database was created. Furthermore, using several major analytical models the shear strength of the FRP materials was computed for each experiment. Finally a comparison was performed between the computed analytical and the reported experimental values.

\section{EXPERIMENTAL DATABASE}

A total of 70 reinforced concrete (RC) beams were used to form the experimental database. All of these RC beams were strengthened using externally bonded inorganic matrix composites. Sixty beams had a rectangular section and ten were T-beams. Sections smaller than $100 \mathrm{~mm}$ by $200 \mathrm{~mm}$ were excluded from the study in order to avoid possible size effects. Although the beams were strengthened with different types of fibers they were all bonded to the concrete using inorganic matrices. All beams that were considered failed due to FRP debonding, FRP delamination or FRP fracture. FRP delamination is a failure mode that is unique to inorganic composites. More specifically it has been reported in some cases that the fibers slip through the matrix. This happens because some fibers are epoxy coated, which is inherently incompatible with inorganic matrices used for impregnation.

Tetta et al. [9] reported experiments on 14 beams of rectangular section (102 by $203 \mathrm{~mm}$ ). Eight of these beams were strengthened with carbon fiber textile reinforced mortar composites using a U-shape, side bonding or fully wrapped configurations, and were included in the experimental database.

Loretto et al. reported on 4 beams of rectangular section (152 by $306 \mathrm{~mm}$ ). The beams were strengthened with fiber reinforced cementitious matrix. They were strengthened using a $\mathrm{U}$-shaped configuration.

Six beams reported by Contamine et al. [10] were included in the database. The beams had a rectangular section $(150$ by $220 \mathrm{~mm})$ and were strengthened with AR Glass using a side bonding or a U-shape configuration. The beams were tested under four point bending.

Larbi et al. [11] reported on a series of tests performed on strengthened RC beams. Five beams were included from their study. Beams were strengthened with metallic and glass fibers using a cementitious mortar. The beams were rectangular with a cross section of 150 by $220 \mathrm{~mm}$. 
Three beams were included from a study conducted by Triantafillou and Papanicolaou [7]. The beams were rectangular $(150$ by $300 \mathrm{~mm})$ and were strengthened using carbon fiber bonded with a cementitious mortar.

Blanksvärd et al. [12] conducted experiments on rectangular beams strengthened with mortar and carbon fibers with different grids. Seven of their beams were included in the database.

Al-Salloum et al. [13] reported experiments on ten strengthened beam. Eight of their specimens were included in the database. The beams were rectangular and were strengthened using basalt fibers.

Tzoura and Triantafillou [14] examined RC T-beams strengthened with either textile reinforced mortars or FRP. The beams had a cross section of 100 by 150 by $350 \mathrm{~mm}$. Some of the specimens were strengthened with anchored composites. A total of 10 beams were included in the database.

Escrig et al. [15] conducted experiments on nine beams strengthened with different types of composites and different inorganic matrices. The beams were rectangular with a cross section of 300 by $300 \mathrm{~mm}$ and were tested under three point bending over a span of $1600 \mathrm{~mm}$. A total of 6 beams from this study were included in the database.

Data from experiments conducted on seven beams reported by Ombres [16] were used in the database. The beams had a rectangular cross section with dimensions 150 by $250 \mathrm{~mm}$, and were strengthened with PBO fiber cement based composite materials in U-shaped configuration.

Finally, Azam and Soudki [17] published an article that presents the results of an experimental study conducted to investigate the effectiveness of different types of fabricreinforced cementitious matrix (FRCM) composite systems to strengthen shear critical RC beams. Seven shear-critical $\mathrm{RC}$ beams were tested. The test variables included the strengthening material (glass FRCM or carbon FRCM) and the strengthening scheme (side bonded or u-wrapped). The beams were rectangular and had a cross section of 150 by $250 \mathrm{~mm}$. A total of six beams were used in the experimental database.

Theoretical predictions of the models vs. experimental values were plotted for each analytical model. As mentioned previously theoretical predictions and experimental values refer to the composite contribution to the total shear capacity. The experimental values of the composite contribution have been calculated by subtracting the values reported from the tests on control beams from the values obtained from FRP strengthened beams, assuming that the difference is the composite contribution to shear strength.

\section{ANALYTICAL MODELS}

All existing models and codes treat the shear contribution of the externally bonded FRP the same way as they treat the stirrup contribution. More specifically, the total shear capacity of the beams is computed by adding the contribution of the concrete $\left(V_{c}\right)$, the contribution of the steel stirrups $\left(V_{s}\right)$ to the contribution of the FRP $\left(V_{f r p}\right)$. In our study since control beams were used in all experiments, in order to minimize errors, we only compared the contribution of the FRP $\left(V_{f r p}\right)$
The following analytical models were used in this study:

\section{A. Khalifa et al. (1998) [18]}

The model was based on two previous studies reported by Triantafyllou [19] and Maeda et al. [20]. The model offered a modified effective stress for all types of failure. In case of rupture the FRP shear strength is provided by Eq. (1):

$$
V_{f}=\frac{A_{f} f_{f e}(\sin \beta+\cos \beta) d_{f}}{s_{f}} \quad[N]
$$

The effective fiber stress is calculated by multiplying the ultimate fiber stress by an effective ratio $(\mathrm{R})$ that is limited to 0.5 . The effective ratio $\mathrm{R}$ can be calculated using Eq. (2)

$$
R=0,5622\left(\rho_{f} E_{f}\right)^{2}-1,2188\left(\rho_{f} E_{f}\right)+0,778 \leq 0,50
$$

Equation (2) was based on a regression analysis of experimental data. Since (1) is not taking in consideration the debonding mechanism, the $R$ factor was derived considering a bond mechanism model.

A different formula (3) was provided in the case of debonding, that took into consideration a bond mechanism model:

$$
V_{f}=\frac{2 L_{e} w_{f} \tau_{b u} w_{f e}}{s_{f}} \quad[N]
$$

where:

$w_{f e}$ is the effective width of the composite that was introduced to control the composite bond:

$w_{f e}=d f$ in case of fully wrap

$w_{f e}=d f$-Le for U-shape bonding

$w_{f e}=d f-2 L e$ for side bonding

$\tau_{b u}$ is the average shear stress that corresponds to debonding and can be calculated using (4):

$$
\tau_{b u}=k\left(\frac{f_{c}^{\prime}}{42}\right)^{\frac{2}{3}} E_{f} t_{f} \quad[\mathrm{MPa}]
$$

In the case of debonding or delamination the model suggests using the FRP's effective length which is:

$$
L_{e}=e^{6,314-0,58 \ln \left(t_{f} E_{f}\right)} \quad[\mathrm{mm}]
$$

Finally the model provides a series of safety factors similar to the ones in ACI and Eurocodes.
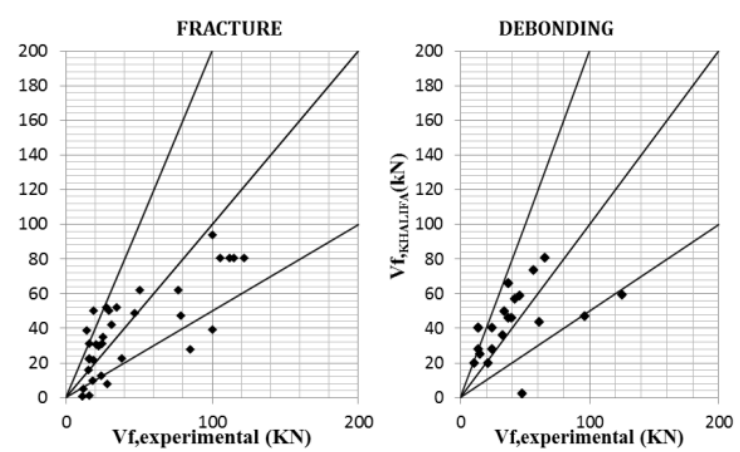

Fig. 1. Experimental vs. Analytical shear strength from the model of Khalifa et al.

Experimental vs. theoretical values were plotted using the analytical equations provided by Khalifa et al. [18] and are shown in Fig. 1. Two separate graphs are provided. The one 
on the left illustrates the performance of beams that exhibited a debonding type failure while the one on the right the performance of beams that failed due to fiber fracture. It should be noted that the term debonding includes all types of premature composite failure such as fiber slippage, composite delamination, horizontal shear cracking of concrete, etc. As can be seen three lines have been drawn in each graph. The one in the middle (diagonal of the graph area) indicates that the experimental values are exactly equal to analytical. The line that begins at the beginning of the axis and reaches the top horizontal axis indicates a fifty percent difference limit. Finally, the other line that ends at the middle of the vertical axis also indicates a $50 \%$ limit. A point above the middle diagonal line means that an analytical value is higher than the corresponding experimental, and therefore one can conclude that the model overestimates the composite shear strength. If the point is between the top and the central diagonal line the difference between the experimental and the analytical values is less than $50 \%$. Similarly, if the point is below the middle line that means that the experimental strength is higher than the analytical and thus the model provides safe predictions of strength. If the point is between the central and the lower line the difference is less than $50 \%$, which is the desirable outcome. Points over the upper line or under the lower line denote differences of more than $50 \%$ between experimental and analytical strengths.

Khalifa's model presented problems in some cases. More specifically, when the depth of the beam was quite large the effective length $L_{e}$ was large and thus the effective width was very small (in some cases even negative). It should be noted that equations for $R$ and $L_{e}$ were results of a regression analysis. Unfortunately at the time the model was developed, the number of available experimental data was limited, and thus the equations need to be adjusted.

An examination of Fig. 1 can lead to the conclusion that the model proposed by Khalifa et al [18] is overestimating the shear strength of the beams for both types of failure. Especially in the case of debonding only five of the tested beams, representing a $25 \%$ of the total number of beams, exhibited an experimental strength higher than that calculated using the model. In the case of fiber fracture only $50 \%$ of the analytical shear strengths were lower than the experimental. It is thus evident that an adjustment of the safety factors is necessary.

\section{B. Triantafillou and Antonopoulos (2000) [21]}

The model is based on truss analogy and differentiates the effective strain of the FRP based on the type of strengthening (fully wrapped FRP or U-shaped) and the FRP's axially rigidity. The effective strain that is being used to determine the mode of failure was shown that is dependent on the axial rigidity of the composite and the effective bond length. The effective strain is used as the minimum of: maximum strain limited due to debonding, strain to control crack opening, and strain corresponding to shear failure related to FRP rupture.

The shear strength provided by the FRP can be calculated using:

$$
V_{f d}=0,9 \frac{\varepsilon_{f k, e}}{\gamma_{f}} E_{f} \rho_{f} b_{w} d(1+\cot \beta) \sin \beta \quad[k N]
$$

In (6) the composite shear strength depends mainly on the characteristic effective strain, $\varepsilon_{f k, e}$, which is equal to $80 \%$ of the effective strain. The effective strain can be calculated with different equations depending on the type of the strengthening method. For fully wrapped beams the effective FRP strain will be:

$$
\varepsilon_{f, e}=0,17\left(\frac{f_{c}^{\frac{2}{3}}}{E_{f} \rho_{f}}\right)^{0,30} \varepsilon_{f, u}
$$

While for side bonded or U-shaped composites the effective strain could be calculated by:

$$
\varepsilon_{f, e}=\min \left[0,65\left(\frac{f_{c}^{\frac{2}{3}}}{E_{f} \rho_{f}}\right)^{0,56} \times 10^{-3}, 0,17\left(\frac{f_{c}^{\frac{2}{3}}}{E_{f} \rho_{f}}\right)^{0,30} \varepsilon_{f, u}\right\rfloor
$$

Equations (7) and (8) where derived based on the finding that fiber stiffness and concrete strength affect the shear strength and the mode of failure of the composite. Nevertheless both equations were based on regression analysis of a relatively limited number of experimental data.

The safety factor $\gamma_{f}$ in (6) is suggested to be taken equal to 1.20 for Carbon fibers and 1.30 for Glass fibers in case of fracture while it should be taken equal to 1.30 regardless of FRP type in the case of delamination.

The results for this model have been plotted in Fig. 2. It can be seen that the model significantly underestimates the strength in case of failure based on composite fracture. Although most of the predictions $(90 \%)$ were on the safe side, approximately $50 \%$ of the predicted values were extremely conservative. Also it is evident, that the higher the experimental shear strength of the composite the greater the difference between experimental and analytical values. On the other hand, for beams that failed due to debonding it can be observed that the predictions fit better to the experimental data since values are closely scattered around the diagonal. However, the model provides less safety, since $50 \%$ of the beams failed at loads lower than the predicted. This is even more evident for beams that exhibited low experimental strengths.
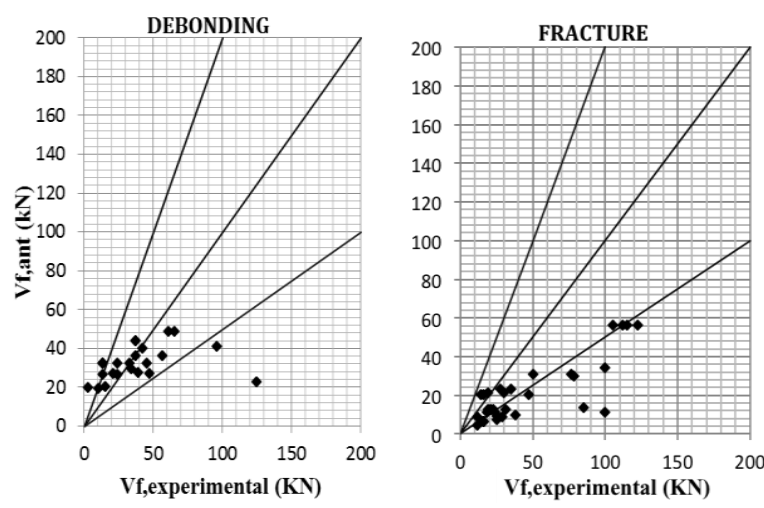

Fig. 2. Experimental vs. Analytical Shear strength for inorganic matrix composites obtained using the model of Triantafillou and Antonopoulos.

\section{Chen \& Teng [22]-[24]}

Chen and Teng provided one of the most detailed shear strength models. The model was a result of an extensive study that started in 2001 [24] and resulted in the final model that was described in two articles [22], [23]. The 
authors created a model based on the truss model theory and used the assumption of non-uniform stress distribution along the shear crack.

The authors provided one general equation (9) to be used to predict the shear strength of the composite:

$$
V_{f r p}=2 f_{f r p, e} t_{f r p} w_{f r p} \frac{h_{f r p, e}(\cot \vartheta+\cot \beta) \sin \beta}{s_{f r p}}[N]
$$

It is noted that Chen and Teng reported two models based on the mode of failure, one for debonding [23] and one for fiber rupture [22]. In the case of FRP rupture, it is mentioned that it will occur when fiber stress reaches:

$$
f_{f r p, e}=D_{f r p} f_{f r p}[M P a]
$$

where $D_{f r p}$ is the stress distribution factor assumed to be equal to:

$$
D_{f r p}=\frac{1+\zeta}{2}
$$

and:

$$
\zeta=\frac{z_{t}}{z_{b}}
$$

where, $z_{t}$ and $z_{b}$ are the top and bottom coordinates of the FRP.

The debonding model developed by Chen and Teng [23] is based on the principle that: "an effective bond length beyond which an extension of the bond length cannot increase the bond strength". The maximum stress in the FRP at debonding is given as:

$$
\sigma_{f r p, \max }=\min \left\{\begin{array}{l}
f_{f r p, e} \\
0,427 \beta_{w} \beta_{L} \sqrt{\frac{E_{f r p} \sqrt{f_{c}^{\prime}}}{t_{f r p}}} \quad[M P a]
\end{array}\right.
$$

Thus, the authors described that the maximum stress in the FRP will be either because of FRP rupture $\left(f_{\text {frp }, e}\right)$ or debonding. The two coefficients $\beta_{L}(14), \beta_{w}$ (15) reflect the effective bond length and the effect of FRP to concrete width ratio, respectively.

$$
\begin{array}{r}
\beta_{L}=\left\{\begin{array}{l}
1 \quad \text { if } \quad \lambda \geq 1 \\
\sin \frac{\pi \lambda}{2} \quad \text { if } \quad \lambda<1
\end{array}\right. \\
\beta_{w}=\sqrt{\frac{2-\frac{w_{f r p}}{s_{f r p} \sin \beta}}{1+\frac{w_{f r p}}{s_{f r p}} \sin \beta}}
\end{array}
$$

The normalized maximum bond length, $\lambda$ (16), the effective bond length, $L_{e}(18)$, and the maximum bond length, $L_{\max }(17)$, are provided by:

$$
\begin{gathered}
\lambda=\frac{L_{\max }}{L_{e}} \\
L_{\max }=\left\{\begin{array}{ll}
\frac{h_{f i p, e}}{\sin \beta} & U-\text { jacket } \\
\frac{h_{f i p, e}}{2 \sin \beta} & \text { side-bonded }
\end{array} \quad[\mathrm{mm}]\right.
\end{gathered}
$$

$$
L_{e}=\sqrt{\frac{E_{f r p} t_{f r p}}{\sqrt{f_{c}^{\prime}}}}[\mathrm{mm}]
$$

Finally, Chen and Teng [23] proposed design guidelines using the previously mentioned methodology and introduced a safety factor $\left(\gamma_{b}=1.25\right)$. They also suggested to use 0.315 instead of 0.427 in (13), and a maximum allowable spacing of FRP strips $S_{f r p}$ :

$$
S_{f r p}-\frac{w_{f r p}}{\sin \beta} \leq \frac{h_{f r p, e}(1+\cot \beta)}{2} \quad \text { and } \quad S_{f r p} \leq 300 \mathrm{~mm}
$$

The spacing was assumed necessary by the authors in order to make sure that in large beams that at least one FRP strip will intersect a shear crack.

The analytical results obtained using this model are shown in Fig. 3 in comparison to the experimental results. It is evident that this model provides better results in terms of safety compared to the ones presented previously. Most of the composite design strengths are lower than the experimental. But while for debonding the differences between experimental and analytical were typically less than $50 \%$, this was not the case for beams that that failed due to composite fracture. For the latter the model underpredicted significantly the shear strength of beams with composite shear strength of more than $50 \mathrm{kN}$ with differences well above $50 \%$. Furthermore, a statistical analysis proved that there is a significant correlation between experimental and theoretical values. Spearman correlation coefficients were calculated as 0.706 for debonding and 0.675 for fracture, proving a very good correlation.
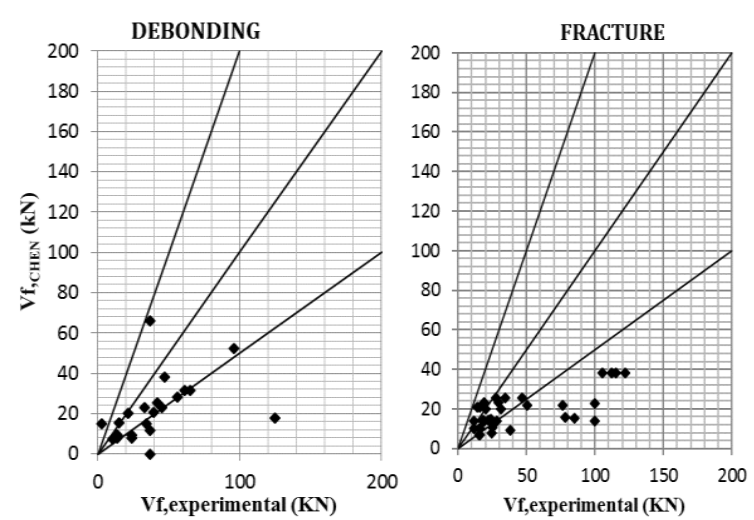

Fig. 3. Experimental vs. Analytical Shear strength for inorganic matrix composites obtained using the Chen and Teng's models.

\section{Zhang and Hsu [25]}

Zhang and Hsu [25] described a model that also includes a reduction factor for the evaluation of the effective strain. In order to calculate the reduction factor formula, when debonding failure occurs, the authors used the initial model proposed by Khalifa et al. [18]. Since more data from test results were available, they used a power regression line to determine the reduction factor, $R$. The power regression resulted in higher R-square values than the polynomial used by Khalifa et al. [18], which led to the conclusion that the power regression line gives a more realistic prediction of the FRP shear strength component. It was observed that the axial rigidity of the composite and the compressive concrete strength affect the fiber effective strain and determine the mode of failure. More specifically they suggested that fiber rupture occurs at $0<\rho_{f r p} E_{f r p}<0.55 \mathrm{GPa}$, while debonding 
occurred at $0.55<\rho_{f r p} E_{f r p}<1.2 \mathrm{GPa}$. Thus, debonding controls the failure mode as the CFRP laminates become thicker and stiffer.

The CFRP shear strength contribution, $V_{f}$, can be calculated as:

$$
V_{f}=\frac{A_{f} E_{f} \varepsilon_{f e}(\sin \beta+\cos \beta) d_{f}}{s_{f}} \quad[k N]
$$

Equation (20) is quite similar to equations in other models. The $V_{f}$ depends mainly on the FRP effective strain $\varepsilon_{f e}$ which in turn can be taken as:

$$
\varepsilon_{f e}=R \varepsilon_{f u}
$$

where $R$ is a reduction factor and can be defined for FRP rupture as:

$$
R=0,1466\left(\rho_{f} E_{f}\right)^{-0,8193}
$$

For FRP debonding the reduction factor $\mathrm{R}$ can be calculated using the formula:

$$
R=1,4871\left(\frac{\rho_{f} E_{f}}{f_{c}^{\prime}}\right)^{-0,7488}
$$

Zhang and Hsu also suggested a second approach in order to calculate the reduction factor $\mathrm{R}$ for debonding failures that was based on a pure shear stress transfer equilibrium:

$$
R=\frac{\tau_{\max } L_{e}}{2 f_{f u} t_{f}} \leq 1
$$

where $\tau \max$ is the shear strength of concrete:

$$
\tau_{\max }=\left(7,64 \times 10^{-4} \times{f^{\prime}{ }_{c}^{2}}^{2}\right)-\left(2,73 \times 10^{-2} \times{f^{\prime}}_{c}\right)+6,38 \quad[M P a]
$$

The FRP effective length, $L_{e}$, was suggested to be taken equal to $75 \mathrm{~mm}$, which may not always the best value.
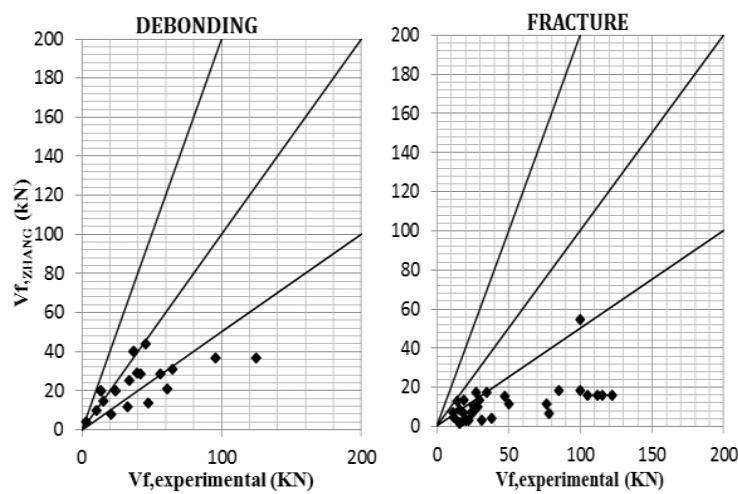

Fig. 4. Experimental vs. Analytical Shear strength for inorganic matrix composites obtained using the Zhang and Hsu's model.

This model is quite similar to the ones presented by Khalifa et al. [18] and Triantafillou and Antonopoulos [21], but provides better results since it is more contemporary and the empirical equations were derived using a larger number of experimental data. The performance of this model is shown in Fig. 4. Experimental values of shear strength contribution are plotted in the horizontal axis and analytical values in the vertical. It is evident that this model leads to safe design strengths for FRP fracture, where $100 \%$ of the experimental values were higher than the design, although in some cases (especially when the strength was high) it underestimates the FRP shear contribution by much more than $50 \%$. The model seems to offer better strength predictions for FRP debonding. Almost all values are within the $\pm 50 \%$ region. Few experimental values were lower than the predicted. Furthermore, a statistical analysis proved that there is a significant correlation between experimental and theoretical values. Spearman correlation coefficient was calculated as 0.668 for debonding and 0.711 for fracture.

\section{E. Carolin \& Täljsten [26]}

The design model proposed by Carolin \& Täljsten is based on the strut and tie model. The authors suggested the use of a calibration factor in order to consider the nonuniform strain distribution over the cross section. This factor, $\eta$, expresses the average strain in the fibers, $\varepsilon_{f}$, over the height of the beam in relation to the strain, $\varepsilon_{\max }$, in the most stressed fiber.

$$
\eta=\frac{\int_{-\frac{h}{2}}^{\frac{h}{2}} \varepsilon_{f}(y) d y}{\left(\varepsilon_{\max } h\right)}
$$

The model uses also an effective strain, $\varepsilon_{f e}$, which is related to the critical strain:

$$
\varepsilon_{f e}=\eta \varepsilon_{c r}
$$

where:

$$
\varepsilon_{c r}=\min \left\{\begin{array}{l}
\varepsilon_{f u} \\
\varepsilon_{b o n d} \\
\varepsilon_{c \max }
\end{array}\right\}
$$

Thus, the model suggests that failure will occur due to fiber fracture, $\varepsilon_{f u}$, debonding, $\varepsilon_{b o n d}$, or cracking of concrete, $\varepsilon_{c m a x}$. The strain $\varepsilon_{c \max }$ is the maximum strain to achieve concrete contribution, and in most cases can be neglected.

For debonding failure the calculation of $\varepsilon_{\text {bond }}$ can be done using the following formulas:

$$
\varepsilon_{\text {bond }}=\frac{1}{E_{f} t_{f}} \sqrt{2 E_{f} t_{f} G_{f}} \begin{cases}\sin \left(\omega L_{b}\right) & , L_{b} \leq L_{c r} \\ 1 & , L_{b}>L_{c r}\end{cases}
$$

where, $L_{b}$, is the development length of the composite fabric:

$$
L_{b}=\left\{\begin{array}{ll}
\frac{d_{f}}{\sin \beta} & , U \text {-Jacket } \\
\frac{d_{f}}{2 \sin \beta} & \text {, side bonding }
\end{array}[\mathrm{mm}]\right.
$$

where $d_{f}$ is the effective depth of the FRP shear reinforcement and $\beta$ is the angle between the horizontal axis and the main fiber direction. $G_{f}$ is concrete's fracture energy given by:

$$
\begin{aligned}
& G_{f} \approx 0,644 f_{c}^{0,19} \quad\left[\begin{array}{lll}
f_{c} & \text { in } & M P a
\end{array}\right] \\
& \omega=\sqrt{\frac{\tau_{\text {max }}^{2}}{2 E_{f} t_{f} G_{f}}} \\
& \tau_{\max }=3,5 f_{c}^{0,19}
\end{aligned}
$$

The shear strength contribution of the fibers can be calculated as: 


$$
V_{f}=\eta \varepsilon_{c r} E_{f} t_{f} r_{f} z \frac{\cos \theta}{\sin \alpha}[k N]
$$

The $r_{f}$ factor in (34) depends on the layout of the strengthening system and is given in (35):

$$
r_{f}= \begin{cases}\sin \beta & \text { for continuous wrapping } \\ \frac{b_{f}}{s_{f}} & \text { for discrete strips }\end{cases}
$$
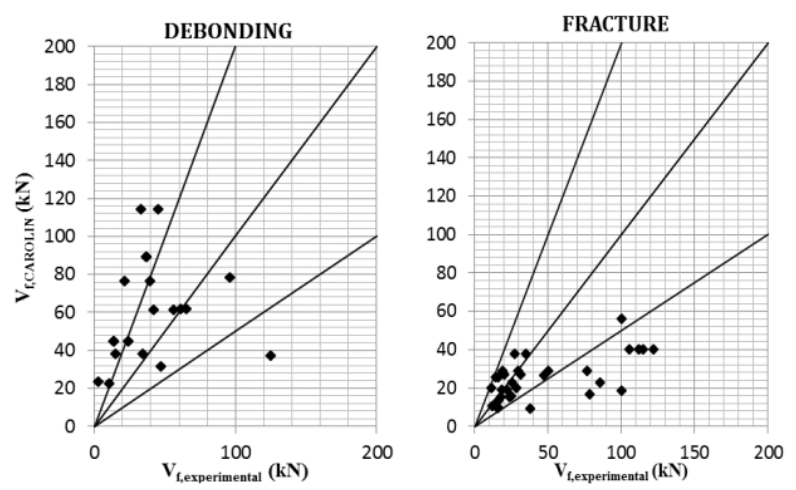

Fig. 5. Experimental vs. Analytical Shear strength for inorganic matrix composites obtained using Carolin \& Täljsten's model.

Results from Carolin \& Täljsten's model are shown in Fig. 5. In case of debonding the model overestimates significantly FRP's contribution to shear strength. Only in $18 \%$ of the strengthened beams the shear contribution of the composite was experimentaly higher than the design value, making the model very unreliable in the case of debonding. In case of fracture failure the model seems to provide better results in terms of safety. Particularly, a safe design was achieved in $75 \%$ of the tested beams However, there is a great variance of the results, with differences between analytical and experimental results of more than $50 \%$ for beams that exhibited an FRP fracture failure of more than $50 \mathrm{kN}$.

\section{F. Monti and Liotta [27]}

A complete design methodology was developed by Monti and Liotta [27] taking in consideration all strengthening schemes and failure modes known at the time. A generalized FRP-concrete bond constitutive law is defined and is used for debonding failure. In order to do so boundary stress limitations are considered, while the stress distribution in the FRP crossing a shear crack is analytically determined.

Furthermore, the following assumptions are considered: the crack depth is equal to the internal lever arm $\mathrm{z}=0.9 \mathrm{~d}$ in the ultimate limit state, the cracks are evenly spaced along the beam axis with an inclination of $\theta$, the resisting shear mechanism is based on the truss analogy for wrapping and U-jacketing. For side bonding, the development of a "crackbridging" resistance mechanism was considered, due to the missing tensile diagonal tie in the truss analogy [27].

This model was the first to account for the concrete corner radius of the beam when $\mathrm{U}$-jacketing and wrapping is used by introducing a reduction coefficient, $\varphi_{R}$, (where $r_{c}$ is the corner radius):

$$
\phi_{R}=0,2+1,6 \frac{r_{c}}{b_{w}}, \quad 0 \leq \frac{r_{c}}{b_{w}} \leq 0,5
$$

The model provides different design equations for the three types of strengthening schemes. The effective bond length $l_{e q}$ (39) and the debonding strength $f_{\text {fed }}$ (38) are defined for side bonding. For side bonding the composite shear strength is:

$$
V_{R d, f}=\frac{1}{\gamma_{R d}} \min \left\{0,9 d, h_{w}\right\} f_{\text {fed }} 2 t_{f} \frac{\sin \beta}{\sin \vartheta} \frac{w_{f}}{p_{f}}[N]
$$

where $f_{f e d}$ is the design fiber stress and is given by:

$$
f_{\text {fed }}=f_{\text {fdd }} \frac{z_{\text {rideq }}}{\min \left\{0,9 d, h_{w}\right\}}\left(1-0,6 \sqrt{\frac{l_{e q}}{z_{\text {rid,eq }}}}\right)^{2} \quad[M P a]
$$

And $l_{e q}$ is the equivalent FRP bond length:

$$
l_{e q}=\frac{s_{f}}{\frac{f_{f d d}}{E_{f}}} \sin \beta[\mathrm{mm}]
$$

In (39) $s_{f}$ is related to slippage of the fibers during debonding and according to the authors should be taken equal to 0.2. For U-shaped FRP configuration the shear component of the fibers and the design FRP stress are:

$$
\begin{gathered}
V_{R d, f}=\frac{1}{\gamma_{R d}} 0,9 d f_{\text {fed }} 2 t_{f}(\cot \vartheta+\cot \beta) \frac{w_{f}}{p_{f}}[N] \\
f_{\text {fed }}=f_{\text {fdd }}\left\lfloor 1-\frac{1}{3} \frac{l_{e} \sin \beta}{\min \left\{0,9 d, h_{w}\right\}}\right\rfloor[M P a]
\end{gathered}
$$

For fully wrapped FRP the equations become:

$$
\begin{gathered}
V_{R d, f}=\frac{1}{\gamma_{R d}} 0,9 d f_{f e d} 2 t_{f}(\cot \vartheta+\cot \beta) \frac{w_{f}}{p_{f}}[N] \\
f_{f c d}=f_{f d d}\left\lfloor 1-\frac{1}{6} \frac{l_{e} \sin \beta}{\min \left\{0,9 d, h_{w}\right\}}\right\rfloor+\frac{1}{2}\left(\varphi_{R} f_{f d}-f_{f d d}\right)\left\lfloor 1-\frac{l_{e} \sin \beta}{\min \left\{0,9 d, h_{w}\right\}}\right\rfloor
\end{gathered}
$$

Monti and Liotta's model is quite balanced in terms of performance for both types of failure as can be seen in Fig. 6 . In terms of safety $85 \%$ of the design strengths are lower than the experimental in case of fracture, and $67 \%$ in case of debonding. The differences between analytical and experimental are relatively small for debonding, with only a few values having a difference of more than $50 \%$. On the contrary the differences between analytical and experimental strengths become larger as the strength increases in the case of inorganic composites fracture. All specimens achieving a composite strength contribution of more than $50 \mathrm{kN}$ have been underestimated by more than $50 \%$.
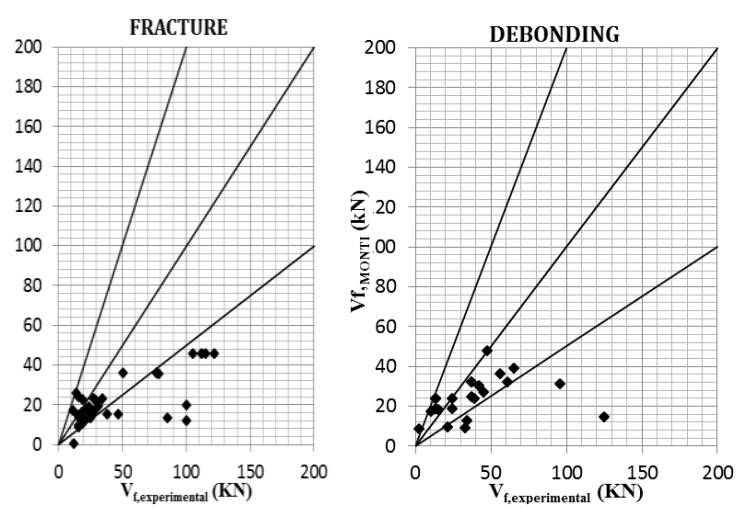

Fig. 6. Experimental vs. Analytical Shear strength for inorganic matrix composites obtained using Monti and Liotta's model. 


\section{CONCLUSIONS}

Predicting the shear resistance of $\mathrm{RC}$ beams strengthened with FRP is still a big challenge for structural engineers. Although many complex models have been developed over the last decade there still is a great discrepancy between analytical and experimental values. Things can become even more complex when different types of matrix or fibers are added to the equation. This paper makes a first attempt, based on the author's knowledge, to investigate if the existing models developed for organic matrix composites can be used for inorganic matrices. From this study the following conclusions can be drawn:

Generally there is a great inconsistency between analytical and experimental values for all models.

Most models significantly underestimate the composite shear strength contribution if failure is dictated by fiber fracture.

Existing analytical/design models could be used to predict the inorganic composite shear contribution but adjustments are necessary to achieve a better correlation between experimental and theoretical results.

The safest model in terms of fiber fracture is Zhang and Hsu's model [25].

The safest model in terms of debonding failure is Chen and Teng's model [23].

Most of the beams used in the experimental database were relatively small compared to real life beams. A size effect may play a role and thus a larger number of specimens is needed in order to evaluate the models' efficiency.

\section{REFERENCES}

[1] S. E. Dritsos, "Jacket retrofitting of reinforced concrete columns," Construction Repair, vol.11, issue 4, pp. 35-40, 1997.

[2] A. Sharif et al., "Strengthening of initially loaded reinforced concrete beams using FRP plates," ACI Structural Journal (American Concrete Institute), vol. 91, issue 2, pp. 160-168, 1994.

[3] P. Balaguru and S. Kurtz. "Use of inorganic polymer-fiber composites for repair and rehabilitation of infrastructures," Maracaibo, Venezuela: ASCE, Reston, VA, USA, pp. 155-168, 1998.

[4] A. Nanni and W. Gold, "Strengthening of RC flexural members with FRP composites," Maracaibo, Venezuela: ASCE, Reston, VA, USA, pp. 144-154, 1998.

[5] H. Toutanji and P. Balaguru, "Durability characteristics of concrete columns wrapped with FRP tow sheets," Journal of Materials in Civil Engineering, vol. 10, issue 1, pp. 52-57, 1998.

[6] C.G. Papakonstantinou and M. F. Petrou, and K. A. Harries, "Fatigue behavior of RC beams strengthened with GFRP sheets," Journal of Composites for Construction, vol.5, issue 4, pp. 246-253, 2001.

[7] T. C. Triantafillou and C. G. Papanicolaou, "Shear strengthening of reinforced concrete members with textile reinforced mortar (TRM) jackets," Materials and Structures, vol. 39, issue 1, pp. 93-103, 2006.

[8] M. Arduini and A. Nanni, "Parametric study of beams with externally bonded FRP reinforcement," ACI Structural Journal, vol. 94, issue 5, pp. 493-501, 1997.

[9] Z. C. Tetta, L. N. Koutas, and D. A. Bournas, "Textile-reinforced mortar (TRM) versus fiber-reinforced polymers (FRP) in shear strengthening of concrete beams," Composites Part B-Engineering, vol.7 7, pp. 338-348, 2015.

[10] R. Contamine, A. S. Larbi, and P. Hamelin, "Identifying the contributing mechanisms of textile reinforced concrete (TRC) in the case of shear repairing damaged and reinforced concrete beams," Engineering Structures, vol. 46, pp. 447-458, 2013.

[11] A. S. Larbi et al., "Shear strengthening of RC beams with textile reinforced concrete (TRC) plate," Construction and Building Materials, vol. 24, issue 10, pp. 1928-1936, 2010
[12] T. Blanksvard, B. Taljsten, and A. Carolin, "Shear strengthening of concrete structures with the use of mineral-based composites," Journal of Composites for Construction, vol. 13, issue 1, pp. 25-34, 2009.

[13] Y. A. Al-Salloum et al., "Experimental and numerical study for the shear strengthening of reinforced concrete beams using textilereinforced mortar," Journal of Composites for Construction, vol. 16, issue 1, pp. 74-90, 2012.

[14] E. T. C. Triantafillou, "Shear strengthening of reinforced concrete Tbeams under cyclic loading with TRM or FRP jackets," Materials and Structures, vol. 49, issue 1, pp. 17-28, 2014.

[15] C. Escrig, L. Gil, E. Bernat-Maso, and F. Puigvert, "Experimental and analytical study of reinforced concrete beams shear strengthened with different types of textile-reinforced mortar," Construction and Building Materials, vol. 83, issue 15, pp. 248-260, 2015.

[16] L. Ombres, "Structural performances of reinforced concrete beams strengthened in shear with a cement based fiber composite material," Composite Structures, vol. 122, pp. 316-329, 2015.

[17] R. S. K. Azam, "FRCM strengthening of shear-critical RC beams," Journal of Composites for Construction, vol. 18, issue 5, 2014.

[18] A. Khalifa, W. Gold et al., "Contribution of externally bonded FRP to shear capacity of RC flexural members," Journal of Composites for Construction, vol. 2, issue 4, pp. 195-202, 1998.

[19] T. C. Triantafillou, "Shear strengthening of reinforced concrete beams using epoxy-bonded FRP composites," Aci Structural Journal, vol. 95, issue 2, pp. 107-115, 1998.

[20] T. Maeda, Y. Asano, Y. Sato, T. Ueda, and Y. Kahuta, "A study on bond mechanism of carbon fiber sheet," in Proc. 3rd Int. Symp. on Non-Metallic (FRP) Reinforced for Concrete Structure, 1997, Japan Con. Inst.: Sapporo, Japan, pp. 279-285.

[21] T. C. Triantafillou and C. P. Antonopoulos, "Design of Concrete Flexural Members Strengthened in Shear with Frp," Journal of Composites for Construction, vol. 4, issue 4, pp. 198-205, 2000.

[22] J. F. Chen and J. G. Teng, "Shear capacity of fiber-reinforced polymer-strengthened reinforced concrete beams: Fiber reinforced polymer rupture," Journal of Structural Engineering-Asce, vol. 129, issue 5, pp. 615-625, 2003.

[23] J. F. Chen and J. G. Teng, "Shear capacity of FRP-strengthened RC beams: FRP debonding," Construction and Building Materials, vol. 17 , issue 1, pp. 27-41, 2003.

[24] J. F. Chen and J. G. Teng, "Anchorage strength models for FRP and steel plates bonded to concrete," Journal of Structural EngineeringAsce, vol. 127, issue 7, pp. 784-791, 2001.

[25] Z. C. Zhang and C. T. T. Hsu, "Shear strengthening of reinforced concrete beams using carbon-fiber-reinforced polymer laminates," Journal of Composites for Construction, vol. 9, issue 2, pp. 158-169, 2005.

[26] A. Carolin and B. Taljsten, "Theoretical study of strengthening for increased shear bearing capacity," Journal of Composites for Construction, vol. 9, issue 6, pp. 497-506, 2005.

[27] G. Monti and M. Liotta, "Tests and design equations for FRPstrengthening in shear," Construction and Building Materials, vol. 21, issue 4, pp. 799-809, 2007.

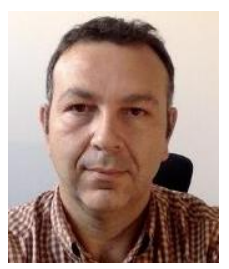

Christos G. Papakonstantinou is an Assistant Professor at the Department of Civil Engineering at the University of Thessaly, Volos, Greece. He received a BSc in civil engineering in 1995 from Aristotle University of Thessaloniki, Greece, an MSc in civil and environmental engineering from the University of South Carolina, Columbia, SC, USA in 2000 and a PhD in civil and environmental engineering in 2003 from Rutgers University, NJ, USA.

He previously worked as an Associate Professor at the University of Massachusetts, Dartmouth, and as faculty of Engineering at Higher Colleges of Technology/Abu Dhabi Men's College. He also has extensive industrial experience in structural design and rehabilitation. His interests include: composite materials, structural rehabilitation, concrete technology and reinforced concrete structures.

Prof. Papakonstantinou is a member of ACI, ASCE and GSCE. 